\title{
Minimising impairment: Protocol for a multicentre randomised controlled trial of upper limb orthoses for children with cerebral palsy
}

Christine Imms ${ }^{1 *}$, Margaret Wallen ${ }^{2}$, Catherine Elliott ${ }^{3}$, Brian Hoare ${ }^{4}$, Melinda Randall ${ }^{1}$, Susan Greaves ${ }^{5}$, Brooke Adair ${ }^{1}$, Elizabeth Bradshaw ${ }^{1}$, Rob Carter ${ }^{6}$, Francesca Orsini ${ }^{7}$, Sophy T. F. Shih ${ }^{6}$ and Dinah Reddihough ${ }^{7}$

\begin{abstract}
Background: Upper limb orthoses are frequently prescribed for children with cerebral palsy (CP) who have muscle overactivity predominantly due to spasticity, with little evidence of long-term effectiveness. Clinical consensus is that orthoses help to preserve range of movement: nevertheless, they can be complex to construct, expensive, uncomfortable and require commitment from parents and children to wear. This protocol paper describes a randomised controlled trial to evaluate whether long-term use of rigid wrist/hand orthoses (WHO) in children with $\mathrm{CP}$, combined with usual multidisciplinary care, can prevent or reduce musculoskeletal impairments, including muscle stiffness/tone and loss of movement range, compared to usual multidisciplinary care alone.

Methods/design: This pragmatic, multicentre, assessor-blinded randomised controlled trial with economic analysis will recruit 194 children with CP, aged 5-15 years, who present with flexor muscle stiffness of the wrist and/or fingers/thumb (Modified Ashworth Scale score $\geq 1$ ). Children, recruited from treatment centres in Victoria, New South Wales and Western Australia, will be randomised to groups (1:1 allocation) using concealed procedures. All children will receive care typically provided by their treating organisation. The treatment group will receive a custom-made serially adjustable rigid WHO, prescribed for $6 \mathrm{~h}$ nightly (or daily) to wear for 3 years. An application developed for mobile devices will monitor WHO wearing time and adverse events. The control group will not receive a WHO, and will cease wearing one if previously prescribed. Outcomes will be measured 6 monthly over a period of 3 years. The primary outcome is passive range of wrist extension, measured with fingers extended using a goniometer at 3 years. Secondary outcomes include muscle stiffness, spasticity, pain, grip strength and hand deformity. Activity, participation, quality of life, cost and cost-effectiveness will also be assessed.
\end{abstract}

Discussion: This study will provide evidence to inform clinicians, services, funding agencies and parents/carers of children with CP whether the provision of a rigid WHO to reduce upper limb impairment, in combination with usual multidisciplinary care, is worth the effort and costs.

Trial registration: ANZ Clinical Trials Registry: U1111-1164-0572.

Keywords: Upper extremity, Splint, Orthosis, Children, Cerebral palsy, Occupational therapy, Intervention, Randomised trial, Cost-effectiveness

\footnotetext{
* Correspondence: Christine.imms@acu.edu.au

${ }^{1}$ Centre for Disability and Development Research, Faculty of Health Sciences,

Australian Catholic University, 17 Young Street, Fitzroy, VIC 3065, Australia

Full list of author information is available at the end of the article
} 


\section{Background}

Cerebral palsy $(\mathrm{CP})$, the most common physical disability in childhood, is a group of disorders of the development of movement and posture that occur as a result of disturbances in the foetal or infant brain [1]. The motor impairment may be accompanied by co-morbidities, including epilepsy, vision or hearing loss, intellectual disability, disorders of communication, behavioural difficulties, and secondary musculoskeletal problems [1]. The most common motor disorder in CP is spasticity, occurring in $86 \%$ of individuals [2]. Spasticity is a velocity-dependent increase in the tonic stretch reflex, with exaggerated tendon reflexes [3] and is characterised by slow, effortful movement [4].

This research is embedded within an International Classification of Functioning, Disability and Health (ICF) framework [5] that articulates a dynamic interaction between impairments at body structure and function level, activity performance and participation. At the body function level, muscle over-activity as result of spasticity and/or dystonia plays a significant role in the development of secondary musculoskeletal impairments in the upper limbs [6] that are common in CP. Secondary impairments include muscle stiffness, loss of active range of movement, joint contracture and pain. Diminished skeletal muscle growth is a key feature in the aetiology of contracture and deformity [6]. Persistent over-activity of skeletal muscle, and subsequent maintenance of a shortened position, can cause a failure of longitudinal muscle growth and muscle adaptation, including increased resistance to passive stretch or stiffness [6, 7]. Subsequently there is a biomechanical imbalance of bone to muscle, as bone continues to grow and muscle growth is impeded [7]. The combined impact of these factors can result in soft tissue retraction, loss of active and passive range of motion and joint contracture $[6,8]$.

Progressive changes in muscle length and stiffening of joints in the upper limbs can ultimately result in a limited ability to reach, grasp and manipulate objects or, in some individuals, a complete lack of functional use of the hands. Strong correlations exist between the degree of upper limb deformity and activity performance [9]. When combined with neurological dysfunction, upperlimb musculoskeletal impairments significantly impact on the ability of children to use their hands to perform daily activities, attain age appropriate independence and develop the autonomy and skills required to participate in activities of importance in home, school and community environments $[10,11]$.

Children with CP are not born with musculoskeletal impairments. There is evidence however, that in children with spastic motor types, these impairments begin to manifest prior to three years of age [12] and that increasing stiffness and progressive loss of range of movement occurs throughout childhood and adolescence [13-15].
A range of treatment options are available for children with $\mathrm{CP}$ that specifically focus on improving hand use. These include activity-based interventions such as goaldirected training, intensive bimanual therapy, modified constraint-induced movement therapy and home programs. Each of these interventions aim to achieve child/ parent-focused goals, and has high-level evidence supporting their effectiveness for increasing activity-level performance and goal achievement [16]. Little evidence is available however, about whether activity-level interventions improve range of movement and reduce secondary musculoskeletal impairments. In addition to activity-based therapies, injection of Botulinum toxin A (BoNT-A) into overactive muscle groups is known to reduce muscle overactivity and has been associated with improved range of movement during a period of chemical denervation, therefore enhancing the effects of upper extremity therapy and the potential for goal achievement and activity performance [17]. Nevertheless, BoNT-A alone in the upper limbs has been shown to have little sustained effect on range of movement [17]. Upper limb surgery is also available to correct deformity once present, although outcomes are variable [18].

Removable orthoses (also called splints) are applied to the forearms, wrists and hands with the goal of either maintaining muscle length and joint range of movement through sustained stretch, or enhancing functional performance. Although orthoses are commonly integrated into intervention strategies with children with $\mathrm{CP}$, there is little evidence supporting the use of upper limb orthoses and wide variation in their prescription, manufacture and intended aims [10, 19]. One controlled trial [20] demonstrated improved effect of BoNT-A when combined with static splinting (term used in the trial by Kanellopoulos et al.) in children with CP. A Cochrane systematic review by Katalinic et al. [21], which included adults and children with a broad range of neurological and non-neurological conditions, demonstrated little benefit of stretching for preventing or reducing contractures. The review concluded that the use of interventions that provided a stretch to muscles, such as upper limb orthoses, be ceased [21]. The application of these findings to children with CP however, is limited. Only five of the 35 randomised trials included children. Of these, three studies included children with CP and each of these evaluated the effects of casting (one in the upper and two in the lower limb) as opposed to orthosis wear. A more recent systematic review of the effectiveness of upper limb orthoses for children with $\mathrm{CP}$ found more equivocal evidence than Katalinic et al. and recommended further methodologically strong research be completed to more effectively inform practice [22].

The clinical rationale for providing upper extremity orthoses is multi-faceted, with both short and long term goals. The focus of the current study is on rigid wrist/ 
hand orthoses (WHO). The primary goal of wearing rigid WHO for all children with $\mathrm{CP}$ is to prevent the development of muscle stiffness, maintain the integrity of soft tissues and prevent the development of abnormal postures and long-term deformity. Due to the diverse nature of $\mathrm{CP}$, the secondary goals generally depend on the child's Manual Ability Classification System (MACS) level. The MACS is a five-level system that describes how children use their hands to handle objects during daily activities [23]. For children in MACS levels I to III, who are able to handle objects in daily life, the secondary aims of WHO prescription are to improve or maintain activity performance and participation through maintenance of good posture for functional use of the hand. Children in MACS levels IV and V have little or no functional use of the upper $\operatorname{limb}(\mathrm{s})$ and the aim of wearing of a rigid $\mathrm{WHO}$ is to maintain posture to facilitate ease of care-giving during daily activities such as bathing, dressing and positioning. Wearing of a rigid WHO is also used to prevent complications associated with muscle shortening such as pain and poor palmar skin hygiene.

Application of the results of previous research has been limited by the length of time in which WHO have been applied and evaluated (often $<6$ months). These time frames are often dictated by the cost of implementing a trial. However, orthosis wear is an intervention aiming for long-term benefits (i.e. the reduction of contracture over time), thus clinicians are appropriately reluctant to change practice based on short-term research evidence. A welldesigned large trial with a long intervention and follow-up period is now critical to determine the long-term outcome of this intervention. This 3 year trial has been designed to balance the need for longitudinal evidence with the complexities of attaining prolonged adherence of participants to an intervention within a controlled trial.

Effective and feasible rigid WHO are those where the benefits outweigh the risks associated with the intervention such as client discomfort, potential for skin breakdown, carer burden in maintaining routine application, follow-up appointments for manufacture and adjustment. Provision of WHO should also contribute positively to the long-term goals of children and families in terms of achievement of participation in meaningful activities during childhood and/ or improved ease of caring for children with CP. Costeffective rigid WHO are those where the benefits outweigh the net costs (defined as cost of the intervention minus the cost offsets) and/or where the relationship between the net costs and outcomes is deemed acceptable (i.e. less than a common decision threshold in Australia, such as $<\$ 50,000$ per Quality Adjusted Life Year (QALY)). Affordable rigid WHO are those where the financial costs for materials, construction and monitoring are within the available budget of third party funders and/or parents.
Wrist-hand orthoses interventions, in combination with activity-based therapy, are aimed at maintaining muscle length, strength and balance, which are required for optimum force generation, effective grasp and manipulation [24] and therefore functional use of affected hands in daily activities. The primary aim of this research is, therefore, to evaluate whether use of rigid WHO over 3 years in children with $\mathrm{CP}$, combined with usual multidisciplinary care, can prevent or reduce musculoskeletal impairment including loss of range of movement and muscle stiffness at the wrist, compared to usual multidisciplinary care alone. The impact of WHO wear on pain, activity performance and participation, as well as ease of caregiving for families will be evaluated along with an assessment of cost-effectiveness of the intervention.

\section{Research questions}

In children aged 5 to 15 years with $\mathrm{CP}$, does the provision of a serially adjustable rigid WHO in combination with usual multidisciplinary care, compared to usual multidisciplinary care alone:

1. Reduce body function and structure impairment including contracture, defined as fixed loss of range of movement, or prevent further loss of range of movement at the wrist, and reduce pain and muscle stiffness at three years from the beginning of the study? This is the primary research question.

2. Improve activity performance and/or ease of caregiving at three years from the beginning of the study?

3. Influence participation and quality of life at three years from the beginning of the study?

In children aged $\geq 5$ years with $\mathrm{CP}$ :

4. What are the effects of the interaction between age and provision of the $\mathrm{WHO}$ plus usual care, and the interaction between severity and provision of the WHO plus usual care, compared to usual care alone, in reducing or preventing further loss of range of movement, muscle stiffness or pain, or improving activity performance or ease of caregiving, at three years from the beginning of the study?

5. What are the incremental costs and potential cost offsets of adding a rigid WHO to usual care compared with usual care alone?

6. From a health sector perspective, what is the costeffectiveness of providing a rigid WHO combined with usual care, compared to usual care alone?

\section{Trial registration}

This study has been registered with the Australian and New Zealand Clinical Trials Registry: U1111-1164-0572. Table 1 displays key registration data. 
Table 1 Minimising impairment trial registration data: Protocol Version 3: 30.10 .2014

\begin{tabular}{|c|c|}
\hline Data Category & Information \\
\hline Primary registry and trial identification number & ANZ Clinical Trials Register: U1111-1164-0572 \\
\hline Date of registration in primary registry & 5.12 .2014 \\
\hline Secondary identification numbers & N/A \\
\hline Sources of money or material support & Australian Catholic University; National Health and Medical Research Council, Australia \\
\hline Primary Sponsor & Investigator led: Professor Christine Imms \\
\hline Contact for public queries & Dr Melinda Randall: Melinda.randall@acu.edu.au \\
\hline Contact for scientific queries & Prof Christine Imms: Christine.imms@acu.edu.au \\
\hline Public title & $\begin{array}{l}\text { Minimising impairment: a multicentre randomised controlled trial of upper limb orthoses } \\
\text { for children with cerebral palsy. }\end{array}$ \\
\hline Scientific title & $\begin{array}{l}\text { Does wearing a rigid upper limb wrist hand orthosis in combination with evidence informed } \\
\text { occupational therapy, compared to evidence informed occupational therapy alone, reduce wrist/ } \\
\text { hand impairment and improve activity and participation outcomes in children aged 5-15 years } \\
\text { with cerebral palsy? }\end{array}$ \\
\hline Countries of recruitment & Australia \\
\hline Health condition studied & Cerebral palsy \\
\hline \multirow[t]{3}{*}{ Intervention } & $\begin{array}{l}\text { Intervention: custom-made serially adjustable rigid wrist hand orthoses to maintain the flexor } \\
\text { compartment (muscles of the wrist, fingers and thumb) in a lengthened position to avoid } \\
\text { shortening of the musculo-tendinous unit and other soft tissue. }\end{array}$ \\
\hline & Control: The control group will not receive a rigid wrist/hand orthosis. \\
\hline & $\begin{array}{l}\text { Both groups: will receive care typically provided by their usual treating organisation. Possible } \\
\text { treatments may include developmentally appropriate, goal focused and evidence-informed } \\
\text { occupational therapy, the use of equipment or BoNT-A injections. }\end{array}$ \\
\hline \multirow[t]{3}{*}{ Key inclusion/exclusion criteria } & Ages eligible for study: 5-15 years; Gender eligible: both; \\
\hline & $\begin{array}{l}\text { Inclusion criteria: A confirmed diagnosis of cerebral palsy as recorded in the medical history; } \\
\text { Presence of flexor muscle stiffness - score at least } 1 \text { on the Modified Ashworth Scale during wrist } \\
\text { extension with fingers extended; May or may not already exhibit contracture at the wrist. }\end{array}$ \\
\hline & $\begin{array}{l}\text { Exclusion criteria: upper limb dystonia without the presence of spasticity; an allergy or sensitivity to } \\
\text { the materials used to construct orthoses; if families are unable to access the study site at the } \\
\text { necessary times; and if families identify factors (e.g. child's behaviour) that impact significantly on } \\
\text { their ability to carry out the intervention }\end{array}$ \\
\hline \multirow[t]{3}{*}{ Study type } & Interventional \\
\hline & Allocation: randomised; intervention model: parallel assignment; Masking: Single blind. \\
\hline & Primary purpose: \\
\hline Date of first enrolment & 28.8.2015 \\
\hline Target sample size & 194 \\
\hline Recruitment status & Recruiting \\
\hline Primary outcomes & $\begin{array}{l}\text { Passive range of wrist extension (measured with the fingers extended) measured using a } \\
\text { goniometer at } 3 \text { years; Active range of wrist movement measured using standardised goniometric } \\
\text { measurement and use of clinometer for measures of supination and inertial motion sensors } \\
\text { measures at } 3 \text { years; }\end{array}$ \\
\hline \multirow[t]{5}{*}{ Key secondary outcomes } & Body function outcomes: Muscle tone; muscle spasticity; grip strength; hand deformity and pain. \\
\hline & Activity outcomes: Self-care; Manual ability; Hand speed and dexterity; ease of care. \\
\hline & Participation outcomes: Attendance and involvement in home, school and community activities. \\
\hline & Quality of Life outcomes: Child and parent. \\
\hline & Health economics outcomes: relative cost and cost-effectiveness. \\
\hline
\end{tabular}

\section{Methods}

Design

This study is a pragmatic, prospective, multicentre, assessor-blinded randomised controlled trial (RCT) with economic analysis that aims to measure the effectiveness of the intervention under usual multidisciplinary care conditions [25]. It will incorporate a two-arm, parallel group superiority design and 1:1 allocation of children to wear $\mathrm{WHO}$ and participate in usual multi-disciplinary care or to multidisciplinary care alone. Assessments 
will be completed at baseline, and every 6 months for 3 years.

\section{Participants Child participants}

Eligible children will be aged 5 to 15 years at the time of recruitment; diagnosed with $\mathrm{CP}$ as recorded in their medical history; and present with flexor muscle stiffness of the wrist as indicated by a Modified Ashworth Scale score $\geq 1$ during wrist extension with fingers extended. Children may or may not already exhibit contracture. Parents are required to be able to understand written and spoken English as it is not feasible to translate all study materials into languages other than English.

Prior upper limb surgery is not an exclusion criteria, however, to avoid interfering with surgical outcomes children will only be eligible if they are at least 12 months post-surgery. In addition, children will be excluded from this study if they have upper limb dystonia without the presence of spasticity; an allergy or sensitivity to the materials used to construct orthoses; if families are unable to access the study site at the necessary times; and if families identify factors (e.g. child's behaviour) that impact significantly on their ability to carry out the intervention.

\section{Therapist participants}

Therapists providing the WHO for any child recruited to the study may be occupational therapists or physiotherapists. They will be asked to consent to providing data related to their discipline and experience, and about the design and fabrication of orthoses they provide to recruited child-participants at each occasion of manufacture or adjustment.

\section{Ethics}

Ethical approval has been received from the Australian Catholic University (HREC: 2014317 V), Cerebral Palsy Alliance in NSW (HREC: 214-08-02), Monash Children's Hospital (HREC: 14199B) and The Royal Children's Hospital (HREC: 34280A) in Victoria and the Princess Margaret Hospital in Western Australia (HREC: 2014060). Parents or guardians of all child-participants will provide informed written consent for their child to take part in the study, as well as consent to complete questionnaires. Children aged 12 years or older will be asked to assent to taking part in the trial if they are capable of doing so. Modifications to the protocol will be reported to each HREC, all investigators and noted on the trial registry.

\section{Sample size}

Sample size calculations were performed according to the primary dependent variable: passive range of movement of wrist extension (measured with fingers extended) at 3 years. Sample size calculations were based on data provided by
Rameckers et al. [26] who reported a baseline standard deviation (SD) of $12.4^{\circ}$ of passive wrist extension in a group with hemiplegic CP. Rameckers et al. [26] included a more homogeneous group of children than we intend and, therefore, in the absence of clear evidence in the literature, the variability has been estimated to approximate $22^{\circ}\left(10^{\circ}\right.$ greater than Rameckers et al.'s more homogenous group). Based on a SD of $22^{\circ}$ for the primary outcome, 77 participants per group would be required to detect a $10^{\circ}$ between-group difference in passive range of movement at 36 months with $80 \%$ power and a two-tailed level of significance of 0.05 . A $10^{\circ}$ difference was chosen as differences greater than 5 to $10^{\circ}$ of movement are deemed clinically important [21]. We used data from our previous successfully completed RCTs of upper limb interventions for children with $\mathrm{CP}$ to estimate withdrawal and loss-to-follow-up rates. Prior withdrawal rates ranged from 0 to $10 \%$, with an additional loss-to-follow up in these RCTs ranging from 0 to $3 \%$ over periods of 6 months. We anticipate that lossto-follow up in this 3 year study will be higher than $3 \%$, and thus estimate a $10 \%$ loss. Consequently we predict a combined withdrawal/loss-to-follow-up rate of $20 \%$. Based on these estimates, we aim to recruit 194 children. Of note, children may have both limbs included in the study. The presence of two limbs was not included in the sample estimates but will be used in the analyses. Including both limbs will increase study power; hence the sample size is expected to be conservative if two limbs are included for some children. Randomised trial experience in Australia suggests we will require at least 12 months to recruit the required sample.

\section{Recruitment}

This multicentre trial will be conducted in Victoria (Monash Children's Hospital and The Royal Children's Hospital), Western Australia (Princess Margaret Hospital and The Ability Centre) and New South Wales (Cerebral Palsy Alliance). The treating clinical teams at each trial site will identify potential participants and provide written and verbal information about the study to potential families (see Fig. 1: Study flowchart). Study advertisements, which will include contact details for the project coordinator and the research assistant assigned to respective sites, will be distributed to clinicians and families. Further advertising will be done through site-specific newsletters, websites and social media with an invitation to contact study personnel or treating therapists for more information about the study. Eligibility will be determined through discussion with parents and clinical examination of the child's upper limb/s.

\section{Randomisation}

Once consent and assent (for those children aged 12 years or more) has been obtained by the research assistant, and 


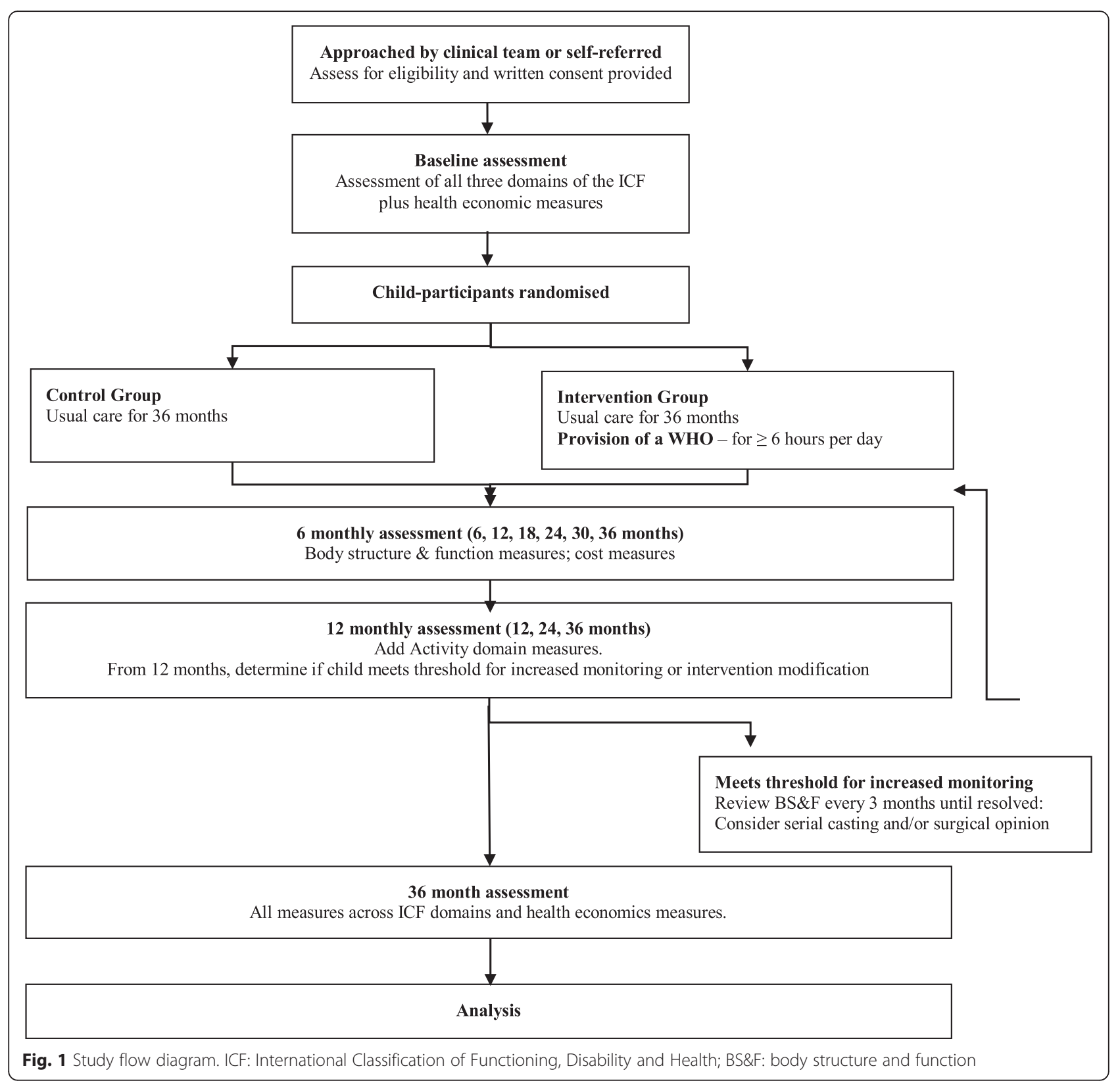

following baseline assessment, children will be randomised to either the treatment or comparison group with an allocation ratio of $1: 1$, using a web-based randomisation procedure to ensure concealed allocation. Randomisation will be completed using randomly permuted blocks of variable length, stratified by study centre ( 5 strata), and by passive wrist range of movement ( 3 strata: wrist extension $>0^{\circ}$ (fingers extended); wrist extension between $-45^{\circ}-0^{\circ}$ (fingers extended); or wrist extension $<-45^{\circ}$ (with or without finger extension)). For children with bilateral involvement, where both limbs meet inclusion criteria, both limbs will be allocated to the same intervention based on the randomisation sequence and stratification will be based on the passive wrist range of the more involved limb. Data will be collected for both upper limbs and included in the analysis at the end of the study for limb-based outcomes.

\section{Interventions}

All children will receive care typically provided by their usual treating organisation. Possible treatments include occupational therapy (e.g. goal-directed training, intensive bimanual therapy), prescription and use of equipment and /or BoNT-A injections. Some children may already have been prescribed a WHO. The WHO of those children allocated to the intervention group will be assessed 
to ensure it meets the intervention protocol, and adjusted if necessary. Those in the control will cease wearing their WHO. Due to the pragmatic nature of this trial, study duration and anticipated heterogeneity of the study sample and possible co-interventions, it will not be possible to standardise co-interventions, however a detailed log will be kept and efficacy/efficiency implications of a non-standardised comparator will be carefully assessed.

\section{Treatment group}

The treatment group will receive a custom-made serially adjustable rigid WHO to maintain the flexor compartment (muscles of the wrist, fingers and thumb) in a lengthened position to avoid shortening of the musculotendinous unit and other soft tissue. For children with unilateral involvement only one hand will be provided with an orthosis. For children with bilateral involvement, both hands will be treated if clinically indicated. The WHO may involve variations of a volar or dorsal/volar orthosis. Volar orthoses are placed on the palmar aspect of the hand and forearm; dorsal/volar orthoses have a component on the palm and a second, linked component on the back of the forearm. Both types of orthoses are designed and fabricated to achieve the same aims. Orthoses will be made of thermoplastic material, which when gently heated loses rigidity allowing it to be conformed to the child's arm in the required position. On cooling, thermoplastics regain rigidity. Straps will be used to maintain the correct position of the child's wrist/hand in the orthosis.

As there is no evidence to guide the optimal position to achieve with orthoses, wearing regimes or follow-up procedures, a national group of expert occupational therapists developed consensus-based guidelines to include in the study operating procedures. Rather than specify muscle and joint positions to achieve with orthoses, these guidelines provide principles to guide individualised positioning of the thumb, fingers and wrist in the orthosis based on the available passive range of motion of the flexor compartment and the relative contribution of wrist and finger flexor stiffness. Briefly, the thumb is positioned midway between full extension and abduction to maintain web space while avoiding metacarpal-phalangeal (MCP) joint hyperextension. Fingers are placed in a small amount of flexion $\left(10-30^{\circ}\right.$ at MCP and inter-phalangeal joints) to prevent the hand slipping in the orthosis. The orthosis is then fabricated to maintain thumb and finger position with the wrist positioned in the orthosis to achieve stretch to the flexor compartment. Each WHO will be fabricated following a clinical assessment of the child's range of motion and muscle tone. Therapists in participating study sites will fabricate the orthoses as part of their usual clinical duties to achieve an individualised position of the wrist and hand according to pre-determined principles outlined in the consensusbased guidelines.

To achieve a prolonged positioning effect, children will be asked to wear the orthosis for a minimum of $6 \mathrm{~h}$ for each $24 \mathrm{~h}$ period of the 3 years of the study [27]. Although night time wear is recommended, it may be appropriate for some children to wear the orthosis during the day: for instance, children with more severe impairments who do not use their hands to carry out activities and therefore for whom wearing a WHO is unlikely to impact on functional hand use. Children with bilateral upper limb involvement where both hands meet the criteria for wearing an orthosis will be asked to wear both orthoses each night. If sleep quality is compromised as a result of concurrently worn bilateral orthoses, recommendations will be to: (i) wear both orthoses each day; or (ii) wear one orthosis at night, the other during the day; or (iii) alternate wear of each orthosis on alternating nights.

The therapists who manufacture the WHO will monitor them in a manner consistent with usual clinical practice. Orthoses will be replaced or serially adjusted over time according to clinical indications to accommodate growth, ensure good fit and maintain appropriate positioning. Serial adjustment involves re-heating the orthosis and remoulding it to achieve an adjusted position. The altered position may be in a position of greater wrist/finger extension in response to improvement in range of motion or reduced muscle stiffness, or in a position of reduced wrist/finger position if range of motion decreases or muscle stiffness increases.

Families will be provided with verbal and written instructions for donning, caring for and cleaning the orthoses; wearing regimes and guidance for facilitating the child to wear orthoses. Consistent with usual clinical practice, families will be asked to contact the therapist who manufactured the WHO if any difficulties with orthosis wear arise such as rash, red marks, bruises, pain or difficulties wearing the orthosis for $6 \mathrm{~h}$ per $24 \mathrm{~h}$ period.

Fidelity of intervention will be considered in three ways. Therapists fabricating orthoses will be educated by study personnel regarding study requirements, orthoses fabrication and wearing principles, which will also be documented in the standard operating procedures provided to each participating therapist. Participating therapists will complete a questionnaire to record details of the design and fabrication of the orthosis and photograph each orthosis on completion of fabrication to provide study personnel with a digital image for evaluating orthosis manufacture against the pre-determined principles. These photographs will also enable assessment of the nature and design of orthoses used in the study. Data related to adjustments made to the WHO over the study period will also be collected. Finally, the frequency and duration that each child wears the orthosis will be 
recorded daily using a custom-designed application for Android and Apple mobile devices known as Therapy App (TherApp) (see Intervention adherence for details).

\section{Control group}

The control group will receive usual multidisciplinary care without a WHO. Prior to consenting, parents will understand that a child entering the study with a prescribed WHO and allocated to the control group will cease wearing it. Parents and children will be advised there is debate about effectiveness; the study will generate evidence to inform future practice. They will also be informed that children in either group could lose ROM during the trial period as this is the natural history of $\mathrm{CP}$, and because of this, their child will be monitored closely throughout the 3 year study period.

\section{Intervention - modifications}

Children in either group could lose range of movement through the trial period as this is the natural history of CP. In addition, the trial will run for an extended period (3 years) during which growth and physical development will influence children's outcomes. Both groups will, therefore, be carefully monitored for loss of range of motion over time.

Children will be monitored for the first 12 months following enrolment according to the study protocol and will not receive any modification to the intervention during this period. Children assessed at or after the 12-month time point who have lost $\geq 30^{\circ}$ passive range of wrist extension or increased by $\geq 2$ categories on the Modified Ashworth Scale, compared to baseline, in either group will be eligible for increased monitoring. This involves reassessment at 3-monthly intervals and may, in consultation with the child's parents and treating team, involve either serial casting to regain range of movement and/or a referral for a surgical opinion. Casting is common clinical practice in children with $\mathrm{CP}$ to achieve increased passive range of motion over the short term [10].

\section{Criteria for withdrawal}

Withdrawal criteria, which apply to all children, have been specified a-priori to ensure child safety, quality of study data, and the ability to answer the research questions. Children who receive a diagnosis other than $\mathrm{CP}$ or their movement disorder evolves to dystonia without the presence of spasticity, will be withdrawn from the study and all data removed from analyses. Children who have a surgical intervention to the included upper limb or more than three episodes of casting as a modification to treatment during the course of the study may discontinue study treatment but will be followed until study completion and be included in the statistical analyses where possible.

\section{Concomitant care}

Children may participate in a range of upper limb interventions throughout the study according to usual multidisciplinary care, such as assistive technology or equipment and devices, upper limb BoNT-A injections, intensive bimanual therapy, early intervention, goal-directed training, home programs, modified constraint-induced movement therapy, or parent education/coaching [16]. Choice of concomitant interventions will be determined by the child's family and treating team and will be recorded.

\section{Intervention adherence}

Adherence to the allocated intervention will be recorded using TherApp, which is the equivalent to an electronic diary. TherApp will be used in conjunction with a studyspecific questionnaire to assist with measurement of WHO adherence, type of co-interventions and incidence of side effects from orthosis wear. Families of children with $\mathrm{CP}$ were consulted about the content, feasibility and implementation of TherApp.

Group allocation (concealed from the assessors) and personal details will be programmed into TherApp at the time of randomisation to ensure appropriate questions are asked about each child. Parents nominate the time for a daily and/or weekly reminder to be sent to their phone or device. For children in the intervention group, TherApp sends a daily prompt to parents requesting information on daily wearing duration and a weekly request for details on adverse events due to wearing orthoses, or any difficulties the child or family experience with wearing orthoses. TherApp will prompt parents to contact their child's treating therapist if needed. We anticipate this will facilitate adherence to the provision of the $\mathrm{WHO}$ and contribute to treatment fidelity. If TherApp registers no response to the daily prompt, an automated email alert to the research assistant enables appropriate follow up. Parents of children in both groups will also receive a weekly TherApp request to record upper limb therapy received in the previous week. Data recorded on TherApp will be extracted prior to each assessment period and supplemented with 6-monthly parent interviews to record additional details about concomitant therapy in the preceding period.

In addition to the TherApp, a novel pressure sensor has been engineered to record the time orthoses are worn. Three sensors will be implanted in the contactsurface of the orthosis (under the thumb, fingers and wrist). The sensors developed for this study use wireless, blue tooth technology and have a sampling rate of $1 \mathrm{~Hz}$ per second. This tiny device is printed on material safe for human consumption using 3-Dimensional printing technology to meet the combined requirements of accurate measurement, protection of skin integrity associated with device pressure and protection of the child from swallowing a small item. Data obtained from the 
sensor will be used to validate parent-report data extracted from the TherApp in a subset of participants.

\section{Data collection and analyses} Assessors and details of blinding

Assessors will be occupational therapists or physiotherapists blinded to the treatment group of the child. Each assessor will be trained by a chief investigator in reliable administration of all measures and provided with a study assessment protocol to ensure consistency of assessment techniques between assessors. Multiple efforts will be made to retain blinded status of assessors. Treating therapists, research personnel, child-participants and families will be routinely reminded that it is critical that blinding of the assessor is retained and the assessor will remind the family of this on initial contact at each assessment. Study numbers allocated to children will not contain a fixed code denoting intervention group. The success of blinding will not be measured as methodological experts argue against such practice [28]. Therapists providing the WHO are unable to be blinded to treatment group and hence may adjust co-interventions differently in the two groups. Measurement of co-interventions and comparisons between groups is therefore is an important part of this trial. Blinding of parents and participants to intervention group is not possible.

\section{Demographic and diagnostic characteristics}

Consistent with best practice in CP [1], the severity of $\mathrm{CP}$ will be assessed and classified at baseline using the Gross Motor Function Classification System [29], Manual Ability Classification System [23], Communication Function Classification System [30] and Bimanual Fine Motor Function scale [31]. In addition, the type of movement disorder (that is, spastic or mixed) and severity of spasticity in the included upper $\operatorname{limb}(\mathrm{s})$ will be rated using the Hypertonia Assessment Tool [32] and the Australian Spasticity Assessment Scale [33, 34] respectively. Information from these tools will help to describe the characteristics of the study sample and be used in post-hoc analyses of outcomes. A study specific questionnaire will measure a range of demographic and other variables including age, gender, associated conditions (intellectual disability, sensory impairments), family configuration, range of services received and socio-economic status as defined using the Socio-Economic Index for Areas data [35].

\section{Outcome measures}

The primary outcome measure is passive range of wrist extension (measured with the fingers extended). Range of wrist movement is operationalised from $-70^{\circ}$ (full wrist flexion) to $+80^{\circ}$ (full wrist extension) where $0^{\circ}$ indicates a neutral position. Range of movement will be measured using a goniometer for extension/flexion movements, an inclinometer for supination/pronation and inertial motion sensors. Inertial motion sensors, constructed specifically for the trial, will be used to measure active and passive wrist extension, with fingers extended as well as functional range of wrist extension elicited during standardised tasks. Other measures across the domains of the ICF will also be used to evaluate the effect of the intervention. Table 2 displays each variable, the measurement tool selected and provides an overview of psychometric evidence for the selected tools.

\section{Economic analysis}

Economic analysis in the context of trials is designed to answer one or both of two questions: i) does the treatment being evaluated offer value-for-money (i.e. 'allocative efficiency'); and ii) if so, how best to design/implement it (i.e. 'technical efficiency'). In this trial we are focussed on technical efficiency. Specifically, is the care pathway more cost-effective with the addition of a WHO for children with CP than without? Economic methods have been chosen therefore to focus on appraisal using trial-based data (with limited economic modelling) and to deal with variability in usual multidisciplinary care. A comprehensive analysis of usual care activities will enable: i) specification of a weighted average usual care pathway (i.e. weighted by activity prevalence); ii) incremental cost-effectiveness ratios (ICERs) presented by state/site, as well as by overall trial results; and iii) extensive sensitivity/uncertainty analyses to detail cost and outcome drivers.

The technical efficiency focus will make cost-effectiveness analysis (CEA) and cost consequences analysis (CCA) the primary analysis, with ICERs that focus on body function, activity measures and child/family quality of life and health utility outcomes from both health sector and service funder perspectives. The key trial-based ICERs are: i) the 'net cost per $10^{\circ}$ improvement in passive range of movement at 3 years'; ii) the 'net cost per unit of improvement on the Cerebral Palsy-Quality of Life' measure; iii) the 'net cost per Adult Quality of Life-8 Dimensions' [36] improvement for parent/carer; and iv) the 'net cost per Child Health Utility 9D' [37] improvements for children. While economic quality of life instruments are usually focussed on value-for-money comparisons, the difficulties in modelling longer term outcomes in this trial, leads to their primary purpose being to help establish technical efficiency. ICERs will be reported as both point and range estimates. In the CCA, ICERs will be reported and interpreted alongside the full range of body function and activity measures collected. A CostUtility Analysis (CUA) with variable time horizons and best available data will be included in sensitivity analysis against a specified decision threshold (i.e. $<\$ 50,000$ per QALY). In addition to the CEA/CCA and CUA, a broader economic approach will be undertaken to capture policy 
Table 2 Variables and outcome measures

\begin{tabular}{|c|c|}
\hline Variable & Measurement tool \\
\hline Body function: baseline, $6,12,18,24,3$ & 36 months \\
\hline $\begin{array}{l}\text { Passive range of motion: elbow extension, } \\
\text { wrist extension (with fingers extended), wrist } \\
\text { extension (with fingers flexed) supination }\end{array}$ & $\begin{array}{l}\text { Standardised goniometric measurement; } \\
\text { inclinometer for measures of supination; }\end{array}$ \\
\hline
\end{tabular}

Inertial Motion sensors.

Active range of movement: elbow extension, Standardised goniometric measurement wrist extension (with fingers extended), wrist and use of inclinometer for measures of extension (with fingers flexed), supination supination.

Inertial Motion sensors

Functional range of wrist extension during standardised tasks.

Muscle stiffness (finger flexors, wrist flexors, pronators and elbow flexors)

Muscle spasticity (finger flexors, wrist flexors, Modified Tardieu Scale [47] pronators and elbow flexors)

Grip strength

Hand deformity

Thumb position

Hand pain
Australian Spasticity Scale [33]

Hand held dynamometer (CITEC)

Modified Ashworth Scale [47]

Neurological Hand Deformity Classification Scale [50]

House Thumb in Palm classification [51]

Study specific questionnaire
Additional information

Goniometric measurements have a high level of intra-rater reliability when measuring passive range of movement in the lower limb in children with CP (ICC >.80) and SEM of $3.5^{\circ}[44,45]$.

Inertial motion sensors (see additional information below) will be used to measure passive wrist extension with fingers extended only.

See additional information above.

Inertial motion sensors (see additional information below) will be used to measure active wrist extension with fingers extended only.

A wireless inertial motion sensor for children has been designed and engineered for this trial to measure wrist flexion/extension movement during functional activity. The sensors use a combination of inertial sensor technologies to provide an accurate estimate of orientation referenced to a fixed frame [46]. Once correctly positioned they wirelessly capture movement with $3^{\circ}$ of freedom in a virtual reality environment to provide continuous kinematic data during unrestricted functional movements. The validity and reliability of the newly developed sensor has been assessed with 10 children with CP (aged 4-12 years) against 3DMA, the 'gold standard' method to quantify movement. Preliminary data demonstrates the inertial motion sensors have excellent static and dynamic accuracy $\left(+/-0.5\right.$ and $+/-1.2^{\circ}$ respectively).

The six point Modified Ashworth Scale has moderate intra-rater reliability when assessing the elbow (ICC 0.66) and wrist flexors (ICC 0.57) in children with CP [48].

The Modified Tardieu Scale has moderate to high intra-rater reliability when assessing the elbow (ICC 0.65) and wrist flexors (ICC 0.92) in children with CP [48].

The Australian Spasticity Assessment Scale has demonstrated moderate to high inter-rater agreement (47-100 \%) [33]

Dynamometery has been shown to have excellent levels of inter-rater (ICC 0.95) and test-re-test reliability (ICC 0.96) when measuring strength in the hand of children with hemiplegic CP [49].

The Neurological Hand Deformity Classification has evidence of reliability for children with spastic cerebral palsy with high inter-rater agreement (Kappa 0.87) and intra-rater agreement (Kappa 0.91) [15]

This measure has been developed for children with CP based on the predictors of surgical success and has been found to be reliable: Kappa $=0.73$ (rater agreement) and 0.74 (test-re-test agreement) $[49,52]$.

The study specific questionnaire was developed for this study to document parent perception of domains unable to be captured in existing measures. Questions will be completed by the child where possible or by a parent/ carer proxy. Although proxy respondents are known to underestimate pain, parent-reported pain will be required for children who are more severely cognitively impaired or unable to communicate their pain effectively. 
Table 2 Variables and outcome measures (Continued)

\begin{tabular}{|c|c|c|}
\hline \multicolumn{3}{|c|}{ Activity domain of the ICF: baseline, 12, 24 \& 36 months } \\
\hline Self-care skills & $\begin{array}{l}\text { Pediatric Evaluation of Disability } \\
\text { Inventory - Computer Adaptive Test [53] }\end{array}$ & $\begin{array}{l}\text { This is a standardised assessment of how children with } \\
\text { impairments function in the context of their daily life. } \\
\text { The Pediatric Evaluation of Disability- Computer Aided Test } \\
\text { provides an accurate and precise assessment of abilities in } \\
\text { four functional domains (ICC 0.99). For this trial only data } \\
\text { from the Daily Activities domain will be collected. }\end{array}$ \\
\hline Manual ability & ABILHAND-Kids [54] & $\begin{array}{l}\text { This tool has been Rasch analysed and has demonstrated } \\
\text { validity and appropriate range and measurement precision } \\
\text { for clinical practice and research: reliability: } R=0.94 \text {; } \\
\text { reproducibility over time: } R=0.91 \text { [54]. }\end{array}$ \\
\hline Speed and dexterity & Box and Blocks Test [55] & $\begin{array}{l}\text { This test has a high level of intra-rater (ICC 0.99) and } \\
\text { test-retest reliability (ICC 0.85) [56]. }\end{array}$ \\
\hline Hand function & Modified House Scale [57] & $\begin{array}{l}\text { This scale is reliable in children with CP: inter rater reliability } \\
\text { (ICC 0.94-0.96); intra rater reliability (ICC 0.93-0.96) [57]. } \\
\text { Rasch analysis was performed on the original scale and the } \\
\text { items reduced: analysis suggests that the modified version } \\
\text { demonstrates good construct validity [58]. }\end{array}$ \\
\hline Ease of care-giving & Study specific questionnaire & $\begin{array}{l}\text { Parent response to specific questions regarding the child's } \\
\text { ability to use their hands in self-care tasks or, for children } \\
\text { with severe forms of cerebral palsy the ease with which } \\
\text { parents or carer's can complete daily tasks of care for them. }\end{array}$ \\
\hline \multicolumn{3}{|c|}{ Participation domain of ICF: Baseline $\& 3$ years only } \\
\hline Participation & $\begin{array}{l}\text { Participation and Environment } \\
\text { Measure-Child \& Youth [59] }\end{array}$ & $\begin{array}{l}\text { Designed to measure frequency of participation, } \\
\text { involvement during participation and the impact of the } \\
\text { environment on participation in children aged } 5 \text { to } 17 \text { years } \\
\text { [59]. This measure captures participation outcomes in } \\
\text { home, school and community contexts. Reliability of the } \\
\text { frequency scales (ICC range 0.58-0.84) and involvement } \\
\text { scales (ICC 0.69-0.76 is moderate to high [59]. }\end{array}$ \\
\hline $\begin{array}{l}\text { Child Health related quality of life and } \\
\text { care-giving burden }\end{array}$ & $\begin{array}{l}\text { Cerebral Palsy Quality of Life } \\
\text { Questionnaire - Child and Teen versions } \\
{[60,61]}\end{array}$ & $\begin{array}{l}\text { Due to the varying ages and abilities of the child- } \\
\text { participants, both parent- and self-report versions of the } \\
\text { Child or Teen CP Quality of Life will be used to measure } \\
\text { quality of life. Test-re-test reliability for the Child version } \\
\text { was high (ranged from ICC } 0.76 \text { to } 0.89 \text { across } 7 \text { scales) } \\
\text { [61], and moderate to high for the Teen version (ICC } 0.57 \\
\text { to 0.88) [60] }\end{array}$ \\
\hline
\end{tabular}

Health economic measures: Baseline, 12, 24, 36 months

Cost Effectiveness Analysis (CEA) Study specific questionnaire

Cost Utility Analysis (CUA)

Cost Consequences Analysis (CCA)
Child Health Utility -9 Dimensions [37]

Assessment of Quality of Life 8

Dimensions [36]
Data on type and number of health professional appointments attended by child in preceding 6-month time period will be utilised for calculation of healthcare cost as well as out of pocket costs to families. Net incremental costs expressed as ICER to meaningful clinical and physical outcomes (e.g. selected from body function domains; activity domains; and the clinical quality of life questionnaire).

Net ICER to the quality of life improvement for children and parents/carers expressed as QALY using an economic MAUI. Where possible the Child Health Utility will be completed along with the parent proxy version. The Child Health Utility has 9 items, takes 2-3 min to complete and covers worry, sadness, pain, tiredness, an noyance, school work, sleep, daily routine and ability to join in activities. The Child Health Utility-9D demonstrated good validity and high levels of agreement with a similar instrument (ICC: 0.742) [62]. The parent measure of quality of life, the Assessment of Quality of Life 8 Dimensions has high reliability (ICC 0.89) [36].

CEA/CUA reported alongside a broader documentation of child \& family relevant outcomes

Note: ICC intraclass correlation coefficient, SEM standard error of measurement, 3DMA three dimensional motion analysis, ICER Incremental Costs Effectiveness

Ratio, MAUI multi-attribute health utility instruments, QALY quality adjusted life year, CEA Cost effectiveness analysis, CUA Cost utility analysis 
and implementation/policy issues (e.g. acceptability to stakeholders, equity impacts, feasibility of implementation, quality of the evidence base) using Assessment of Cost $\boldsymbol{E f}$ fectiveness $(A C E)$ methods. $A C E$ has been used across a series of commissioned and NHMRC-funded projects [38].

Costs will be calculated using pathway analysis to document treatment activity, specify unit prices and estimate costs and potential cost offsets across the study groups. For usual multidisciplinary care, a number of pathways will be constructed and analysed separately as well as a weighted average comparator. Costs associated with the WHO will be assessed by expenditure category (i.e. salaries, overheads, consumables) with economic data collected using a logbook; all other healthcare costs will be assessed by incidence category (i.e. who bears the cost) using available information from sources such as the Medical Benefit Schedule and Pharmaceutical Benefit Scheme. Sensitivity/ uncertainty analyses will be undertaken to investigate the robustness of the ICERs to variations in key cost, pathway and outcome parameters in the trial and across sites.

\section{Data collection methods for child-participants who exit prematurely}

Children may exit prematurely from the study because of voluntary withdrawal or termination of WHO intervention due to harm (e.g. allergic reaction to materials). Participant retention will be supported within the study through provision of routine follow-up and regular feedback on child progress via the TherApp summary report that can be generated by parents throughout the trial. Where possible, all children will be followed to the study end point ( 3 years) so that data are available for analyses. Lack of adherence to the treatment plan will be recorded using TherApp and will not constitute a reason for withdrawal. Reasons for withdrawal from the intervention, or the study, will be recorded to assist with management of missing data and interpretation of results.

\section{Monitoring of harm and adverse events}

No harm or adverse events from orthoses are reported in the literature but are occasionally noted in clinical practice; these are temporary and non-sentinel. Harm arising from the WHO could include the development of pressure areas on the skin, pain, disturbed sleep or behaviour, and skin allergies from specific splint materials while wearing the orthosis, and heat during orthosis fabrication. Children in both groups are at risk of a reduction in joint range of movement as part of the natural course of $\mathrm{CP}$ during growth and development. Adverse events unrelated to the study may also occur and will be adjudicated by the Data Monitoring Committee. Data related to harm and/or adverse events for all children will be collected throughout the study by the therapist who manufactures the WHO (routine follow up), retrospectively by study research assistants (6 monthly) and via TherApp alerts. If the TherApp registers an adverse event an automated email alert to the study research assistant will enable appropriate follow up.

\section{Data management}

Data will be collected using a combination of paper-based and web-based data forms supported by the secure Research Electronic Data capture (REDCap) data management system [39] hosted at the Murdoch Childrens Research Institute. REDCap supports quality control measures including rule-based data entry to reduce data entry errors. In addition, data cleaning will be undertaken. Secure electronic data storage will be undertaken using REDCap, and secure (locked) local storage of original paper-based versions of data collected will occur in accordance with ethically approved procedures for each trial site. Participants will be assigned identification codes on enrolment to the study. These codes will be used during data entry so that data are de-identified during analyses and only aggregated data reported to protect the privacy of participants.

\section{Statistical methods}

The primary analysis will be by intention to treat. Comparison between the intervention and the control groups in the difference from baseline in the passive range of wrist extension (primary outcome) will be presented as the mean difference between the groups and its $95 \%$ confidence interval, obtained using linear regression adjusted for the stratification factors of site and range of passive wrist extension at baseline. The regression model will be fitted using generalised estimating equations (GEE) to allow for the clustering of observations within children for those with both limbs in the study. To explore the effect of the adherence to WHO wearing schedule (i.e. a dose response relationship), a linear regression model will be fitted with compliance to treatment as a predictor and difference in the passive range of wrist extension from baseline to 36 months as the outcome, applied to all study participants. Again this model will be fitted using GEEs to allow for the clustering of limbs within participants. Evidence for an interaction between age and treatment, and between severity (Neurological Hand Deformity Classification) and treatment, will be explored by the inclusion of interaction terms in the linear regression models as well as GEE models. The analyses will be repeated, adjusting for potential confounders including occasions of upper limb BoNT-A injections and frequency of upper limb intervention. Analysis will also be undertaken using the 'per protocol' population excluding children who received surgical intervention or casting during the study. All data available from children who are withdrawn from the study prior to study completion will be used for 
analysis. Imputation of missing data will only be considered in the primary analysis if less than $10-20 \%$ of the primary outcome is missing and will be undertaken throughout multiple imputation models.

Depending on whether data are continuous, categorical or dichotomous, the appropriate generalized linear model will be used to estimate the effect of treatment across the study period on secondary outcomes, again fitted using generalised estimating equations. All analyses will be adjusted for the same stratification factors as for the primary analysis and carried out on intention to treat and per protocol populations.

\section{Trial governance}

A clinical trials agreement is in place between ACU and each trial implementation site that indicates joint intellectual property. A Steering Committee, which includes two parent advisors and all chief investigators, will ensure the study is completed according to the protocol, ethical standards and established timeframes. In addition, the Steering committee will undertake management of the evaluation of the trial and be responsible for establishing a dissemination plan, including peer reviewed publications. Dissemination activities, including attribution of authorship will be undertaken in accordance with the Australian Code for the Responsible Conduct of Research [40]. There are no publication restrictions. The trial Management Committee, based at ACU in Victoria will oversee and manage the day to day operations of the study. State-based advisory groups in Victoria, New South Wales and Western Australia will undertake state-specific implementation. An independent three-person Data Monitoring Committee will review, based on the Damocles Charter, safety, efficacy, participant retention and protocol compliance data and advise regarding protocol variation [11].

\section{Trial status}

Ethical approval has been received from each participating site; study operating procedures and data collection methods have been finalised; and staff recruited and trained in reliable data collection. Recruitment commenced in 2015 and will be ongoing through 2016 .

\section{Discussion/conclusion}

Hand dysfunction and deformity are prevalent in CP: $85 \%$ of children have spasticity that impacts upper limb structure and function, $\geq 62 \%$ have wrist flexion deformities, and early onset is common [41]. Strong positive correlations exist between hand posture and function [9, 41]. Hand orthoses are time-consuming to make and are challenging for families to implement and for children to wear, but if they prevent deformity and improve hand function, they are a vital treatment. This RCT should provide high quality evidence to resolve the long debate about the value of $\mathrm{WHO}$ and the specific impact of wrist impairment on activity. In addition, three novel measurement devices will be designed and/or engineered, tested and validated in children within the conduct of this trial: (i) TherApp; (ii) within-orthosis tactile sensors; and (iii) inertial motion sensors. The further application of these devices in a diverse range of clinical and research contexts will constitute a significant intellectual and practical contribution to the health sciences. TherApp has potential for application to support data collection in other health intervention research trials and in clinical practice to support the implementation of interventions and facilitate communication between clients and clinicians. Inertial motion sensors have potential applicability to other interventions focused on outcomes at the body structure and function level of the ICF, such as BoNT-A, and the tactile sensors may also provide data about orthosis fit as well as wearing time, if placed within the orthosis at key points of hand-orthosis contact.

The annual cost of $\mathrm{CP}$ in Australia is approximately $\$ 1.5$ billion (0.14 \% of GDP) [42]. Lost wellbeing (as a result of disability and premature death) can be valued at a further $\$ 2.4$ billion [42]. This research will provide Level II (RCT) evidence [43] to inform clinicians, health services, government funding bodies and parents and carers of children with $\mathrm{CP}$ whether the provision of orthoses to prevent upper limb impairment is worth the effort and associated costs. This multicentre RCT along with a companion RCT to be implemented with young children under the age of 3 years will provide high quality evidence of the medium-term effect of rigid upper limb orthoses in children with CP. The second trial aims to determine whether provision of a rigid WHO can prevent the occurrence of contracture and deformity in children aged less than 3 years at time of recruitment. By combining the use of rigid orthoses with usual multidisciplinary therapies, these two trials will investigate a combined intervention more reflective of current best practice than has been previously investigated. The results will provide evidence as to whether the use of rigid upper limb orthoses are needed, or if activitybased therapy alone is sufficient to restore and prevent musculoskeletal impairment in children and adolescents with CP.

\section{Abbreviations \\ ACU: Australian Catholic University; BoNT-A: Botulinum toxin-A; CCA: Cost consequence analysis; CEA: Cost effectiveness analysis; CP: Cerebral Palsy; CUA: Cost utility analysis; GEE: General estimating equations; MACS: Manual Ability Classification System; NHMRC: National Health and Medical Research Council, Australia; QALY: Quality adjusted life years; RCT: Randomised controlled trial; SD: Standard deviation; WHO: Wrist hand orthoses.}

Competing interests

The authors declare they have no competing interests. 


\section{Authors' contributions}

$\mathrm{Cl}, \mathrm{MW}, \mathrm{CE}, \mathrm{BH}, \mathrm{SG}, \mathrm{MR}$ and $\mathrm{BA}$ conceived the study and initiated the study design and implementation. All authors collaborated on the development and refinement of the study protocol and have read and approved the final version. MR is the National Project Coordinator and is based in Victoria. MW will take responsibility for the study implementation in New South Wales. CE will take responsibility for study implementation in Western Australia. BH and SG will take responsibility for study implementation in Victoria. DR is the lead investigator on the CRE-CP which nominated this study as a key area for investigation. RC and SS are responsible for the design, implementation, analysis and interpretation of the health economics component of the study. FO will lead the statistical analysis and data management components. All authors have read and approved the manuscript.

\section{Acknowledgements}

Ms Jacqui Wisemantel and Ms Melissa Weston for their support and expertise as parent advisers to the trial.

Dr Katherine Lee, Centre for Epidemiology and Biostatistics at the Murdoch Childrens Research Institute for support with statistical elements of the trial. Mr Simon Garbellini for his contribution to the development of the consensus based guidelines for WHO prescription.

Dr lain Murray, Mr Weiyang Xu, Dr Cesar Ortega-Sanchez, Department of Electrical and Computer Engineering, Curtin University and Dr Sian Williams, Dr Tifffany Grisbrook and Corrin Walmsley, School of Physiotherapy and Exercise Science, Curtin University for the development of the inertial motion sensors.

\section{Financial/material support}

This clinical trial is funded through a 5 year grant from ACU (\#2013000413) in which $\mathrm{Cl}, \mathrm{MW}, \mathrm{BH}, \mathrm{CE}, \mathrm{SG}, \mathrm{MR}, \mathrm{BA}, \mathrm{EB}, \mathrm{DR}$ are grant holders. Funding received from $A C U$ will support the direct research costs and research assistant salaries. In addition, the study is embedded in a Centre for Research Excellence-Cerebral Palsy (CRE-CP), funded by the NHMRC (APP1057997), in which DR, Cl and RC are chief investigators. The CRE-CP will support the studies by providing funding for a Project Coordinator, for expertise associated with conducting the economic analysis led by RC and SS and statistical support to the led by FO. A small grant was obtained from Curtin University to support development of the motion sensors, in which $\mathrm{CE}, \mathrm{Cl}, \mathrm{MR}$ and $\mathrm{BA}$ are grant holders. The authors also acknowledge the substantial in-kind contribution made by each participating organisation in the conduct of the trial: Princess Margaret Hospital, The Ability Centre, Monash Children's Hospital, Cerebral Palsy Alliance and The Royal Children's Hospital.

ACU, Curtin University and the NHMRC had no direct role in the design of this trial and will not have any role during the execution, analyses, data interpretation or decisions regarding submission of results.

\section{Author details}

'Centre for Disability and Development Research, Faculty of Health Sciences, Australian Catholic University, 17 Young Street, Fitzroy, VIC 3065, Australia. ${ }^{2}$ Cerebral Palsy Alliance, PO Box 6427, Frenchs Forest, NSW 2086, Australia. ${ }^{3}$ School of Occupational Therapy and Social Work, Curtin University, Bentley, Australia. ${ }^{4}$ Monash Children's Hospital, Clayton, Australia. ${ }^{5}$ Royal Children's Hospital, Flemington Rd, Parkville 3052, Australia. ${ }^{6}$ Deakin University, Building BC, Room BC3.113, 221 Burwood Highway, Burwood, Australia. ${ }^{7}$ Murdoch Childrens Research Institute, Parkville, Australia.

Received: 14 October 2015 Accepted: 14 May 2016

Published online: 27 May 2016

\section{References}

1. Rosenbaum P, Paneth N, Leviton A, Goldstein M, Bax M, Damiano D, et al. A report: the definition and classification of cerebral palsy April 2006. Dev Med Child Neurol - Suppl. 2007;109:8-14. doi:10.1111/j.1469-8749.2007.tb12610.x.

2. Australian Cerebral Palsy Register Group. Australian cerebral palsy register report 2009: birth years 1993 to 2003. Sydney: Cerebral Palsy Institute; 2009.

3. Lance JW. Symposium synopsis. In: Feldman RG, Young RR, Koella WP, editors. Spasticity: Disordered Motor Control. Chicago: Year Book Medical Publishers; 1980. p. 485-94.

4. Levit K. Chapter 24: Optimizing motor behaviour using the Bobath approach. In: Vining Radomski M, Trombly Latham CA, editors. Occupational therapy for physical dysfunction. 6th ed. Baltimore: Lippincott Williams \& Wilkins; 2008.
5. World Health Organization. International classification of functioning, disability and health: Short version. Geneva: World Health Organization; 2001.

6. Graham HK, Selber P. Musculoskeletal aspects of cerebral palsy. J Bone Joint Surg - Br. 2003;85B(2):157-66. doi:10.1302/0301-620X.85B2.14066.

7. Hof AL. Changes in muscles and tendons due to neural motor disorders: implications for therapeutic intervention. Neural Plast. 2001;8(1-2):71-81. doi:10.1155/NP.2001.71.

8. Sheean G, McGuire JR. Spastic hypertonia and movement disorders: Pathophysiology, clinical presentation, and quantification. Am Acad Phys Med Rehabil. 2009;1:827-33. doi:10.1016/j.pmri.2009.08.002.

9. Arner M, Eliasson AC, Nicklasson S, Sommerstein K, Hägglund G. Hand function in cerebral palsy. Report of 367 children in a population-based longitudinal health care program. J Hand Surg. 2008;33(8):1337-47. doi:10.1016/j.jhsa.2008.02.032.

10. Autti-Rämö I, Suoranta J, Anttila H, Malmivaara A, Mäkelä M. Effectiveness of upper and lower limb casting and orthoses in children with cerebral palsy: An overview of review articles. Am J Phys Med Rehabil. 2006;85(1):89-103. doi:10.1097/01.phm.0000179442.59847.27.

11. A proposed charter for clinical trial data monitoring committees: Helping them to do their job well. Lancet. 2005;365(9460):711-22. doi:10.1016/501406736(05)17965-3.

12. Willerslev-Olsen M, Lorentzen J, Sinkjær T, Nielsen JB. Passive muscle properties are altered in children with cerebral palsy before the age of 3 years and are difficult to distinguish clinically from spasticity. Dev Med Child Neurol. 2013;55(7):617-23. doi:10.1111/dmcn.12124.

13. Nordmark E, Hägglund G, Lauge-Pedersen H, Wagner P, Westbom L. Development of lower limb range of motion from early childhood to adolescence in cerebral palsy: A population-based study. BMC Med. 2009;7:65. doi:10.1186/1741-7015-7-65.

14. Hägglund $\mathrm{G}$, Wagner P. Development of spasticity with age in a total population of children with cerebral palsy. BMC Musculoskelet Disord. 2008;9:150. doi:10.1186/1471-2474-9-150.

15. Georgiades M, Elliott C, Wilton J, Blair E, Blackmore M, Garbellini S. The Neurological Hand Deformity Classification for children with cerebral palsy. Aust Occup Ther J. 2014. doi: 10.1111/1440-1630.12150

16. Novak I, Mclntyre S, Morgan C, Campbell L, Dark L, Morton N, et al. A systematic review of interventions for children with cerebral palsy: State of the evidence. Dev Med Child Neurol. 2013;55(10):885-910. doi:10.1111/dmcn.12246.

17. Hoare BJ, Wallen MA, Imms C, Villanueva E, Rawicki HB, Carey L. Botulinum toxin $A$ as an adjunct to treatment in the management of the upper limb in children with spastic cerebral palsy (UPDATE). Cochrane Database Syst Rev. 2010(1). doi:10.1002/14651858.CD003469.pub4.

18. Smeulders M, Coester A, Kreulen M. Surgical treatment for the thumb-inpalm deformity in patients with cerebral palsy. Cochrane Database Syst Rev. 2005(4). doi:10.1002/14651858.CD004093.pub2.

19. Teplicky R, Law M, Russell D. The effectiveness of casts, orthoses, and splints for children with neurological disorders. Infants Young Child. 2002;15(1):42-50. doi:10.1097/00001 163-200207000-00007.

20. Kanellopoulos AD, Mavrogenis AF, Mitsiokapa EA, Panagopoulos D, Skouteli H, Vrettos SG, et al. Long lasting benefits following the combination of static night upper extremity splinting with botulinum toxin A injections in cerebral palsy children. Eur J Phys Rehabil Med. 2009;45(4):501-6.

21. Katalinic OM, Harvey LA, Herbert RD, Moseley AM, Lannin NA, Schurr K. Stretch for the treatment and prevention of contractures. Cochrane Database Syst Rev. 2010;9:CD007455. doi:10.1002/14651858.CD007455.pub2.

22. Jackman M, Novak I, Lannin N. Effectiveness of hand splints in children with cerebral palsy: A systematic review with meta-analysis. Dev Med Child Neurol. 2013;56(2):138-47. doi:10.1111/dmcn.12205.

23. Eliasson AC, Krumlinde-Sundholm L, Rösblad B, Beckung E, Arner M, Öhrvall AM, et al. The Manual Ability Classification System (MACS) for children with cerebral palsy: Scale development and evidence of validity and reliability. Dev Med Child Neurol. 2006;48(7):549-54. doi:10.1017/S0012162206001162.

24. Wilton JC. Casting, splinting, and physical and occupational therapy of hand deformity and dysfunction in cerebral palsy. Hand Clin. 2003;19:573-84. doi:10.1016/50749-0712(03)00044-1.

25. Zwarenstein M, Treweek S, Gagnier JJ, Altman DG, Tunis S, Haynes B, et al. Improving the reporting of pragmatic trials: an extension of the CONSORT statement. BMJ. 2008:337:a2390. doi:10.1136/bmj.a2390.

26. Rameckers EAA, Speth LAWM, Duysens J, Vles JSH, Smits-Engelsman BCM. Botulinum toxin-A in children with congenital spastic hemiplegia does not improve upper extremity motor-related function over rehabilitation alone: A 
randomized controlled trial. Neurorehabil Neural Repair. 2009;23(3):218-25. doi:10.1177/1545968308326629.

27. Tardieu C, Lespargot A, Tabary C, Bret MD. For how long must the soleus muscle be stretched each day to prevent contracture? Dev Med Child Neurol. 1988;30(1):3-10.

28. Sackett DL. Clinician-trialist rounds: 6. Testing for blindness at the end of your trial is a mug's game. Clin Trials. 2011;8(5):674-6. doi:10.1177/1740774511419685.

29. Palisano R, Rosenbaum P, Walter S, Russell D, Wood E, Galuppi B. Development and reliability of a system to classify gross motor function in children with cerebral palsy. Dev Med Child Neurol. 1997;39(4):214-23. doi:10.1111/j.1469-8749.1997.tb07414.x.

30. Hidecker MJC, Paneth N, Rosenbaum PL, Kent RD, Lillie J, Eulenberg JB, et al. Developing and validating the Communication Function Classification System for individuals with cerebral palsy. Dev Med Child Neurol. 2011;53(8):704-10. doi:10.1111/j.1469-8749.2011.03996.x

31. Beckung E, Hagberg G. Neuroimpairments, activity limitations, and participation restrictions in children with cerebral palsy. Dev Med Child Neurol. 2002;44(5):309-16. doi:10.1017/S0012162201002134.

32. Fehlings D, Switzer L, Jethwa A, Mink J, Macarthur C, Knights $S$ et al. Hypertonia Assessment Tool (HAT): User Manual. Version: June 2010. Toronto, Canada: Bloorview Children's Hospital: 2010

33. Williams N, Love S, Gibson N, Blair E. Reliability of the Australian Spasticity Assessment Scale. Dev Med Child Neurol. 2008;50(s113). doi:10.1111/j.14698749.2008.00004.x.

34. Love S, Gibson N, Smith N, Bear N, Blair E, Australian Cerebral Palsy Register G. Interobserver reliability of the Australian Spasticity Assessment Scale (ASAS). Dev Med Child Neurol. 2016;58 Suppl 2:18-24. doi:10.1111/dmcn.13000.

35. Australian Bureau of Statistics. SEIFA 2001. Canberra: Australian Bureau of Statistics; 2001.http://www.abs.gov.au/AUSSTATS/abs@.nsf/DetailsPage/2033. 0.55.0012011. Accessed 23rd January 2007.

36. Richardson J, lezzi A, Khan MA, Maxwell A. Validity and reliability of the Assessment of Quality of Life (AQoL)-8D multi-attribute utility instrument. The patient. 2014;7(1):85-96. doi:10.1007/s40271-013-0036-x.

37. Stevens K, Ratcliffe J. Measuring and valuing health benefits for economic evaluation and valuing health benefits for economic evaluation in adolescence: an assessment of the practicality and validity of the Child Health Utility 9D in the Australian Adolescent population. Value Health. 2012;15(8):1092-9. doi:10.1016/j.jval.2012.07.011.

38. Carter R, Vos T, Moodie M, Haby M, Magnus A, Mihalopoulos C. Priority setting in health: origins, description and application of the Australian Assessing Cost-Effectiveness initiative. Expert Rev Pharmacoecon Outcomes Res. 2008:8(6):593-617. doi:10.1586/14737167.8.6.593.

39. Harris PA, Taylor R, Thielke R, Payne J, Gonzalez N, Conde JG. Research electronic data capture (REDCap): A metadata-driven methodology and workflow process for providing translational research informatics support. J Biomed Inform. 2009;42(2):377-81.

40. National Health and Medical Research Council, Australian Research Council. Australian code for the responsible conduct of research. In: Australian Research Council, editor. National Health and Medical Research Council. Australia: Australian Government; 2007.

41. Park ES, Sim EG, Rha DW. Effect of upper limb deformities on gross motor and upper limb functions in children with spastic cerebral palsy. Res Dev Disabil. 2011;32(6):2389-97. doi:10.1016/.jidd.2011.07.021.

42. Economics A. The economic impact of cerebral palsy in Australia in 2007. Cerebral Palsy Australia: Sydney; 2008.

43. National Health and Medical Research Council. How to use the evidence: Assessment and application of scientific evidence. In: Government A, editor. Canberra, Australia. 2000. p. 85

44. Herrero P, Carrera P, García E, Gámez-Trullén EM, Oliván-Blázquez B. Reliability of goniometric measurements in children with cerebral palsy: A comparative analysis of universal goniometer and electronic inclinometer. A pilot study. BMC Musculoskelet Disord. 2011;12:155. doi:10.1186/1471-2474-12-155.

45. Horger M. The reliability of goniometric measurements of active and passive wrist motions. Am J Occup Therap. 1990;44(4):342-8. doi:10.1186/1471-2474-12-155.

46. Pérez R, Costa U, Torrent M, Solana J, Opisso E, Cáceres C, et al. Upper limb portable motion analysis system based on inertial technology for neurorehabilitation purposes. Sensors. 2010;10:10733-51. doi:10.3390/s101210733.

47. Boyd RN, Graham HK. Objective measurement of clinical findings in the use of botulinum toxin type A for the management of children with cerebral palsy. Dev Med Child Neurol. 1999;6:S23-35. doi:10.1111/j.1468-1331.1999.tb00031.x.
48. Numanoğlu A, Günel MK. Intraobserver reliability of modified Ashworth scale and modified Tardieu scale in the assessment of spasticity in children with cerebral palsy. Acta OrthopTraumatol Turc. 2012;46(3):196-200. doi:10.3944/AOTT.2012.2697.

49. Klingels K, De Cock P, Molenaers G, Desloovere K, Huenaerts C, Jaspers E, et al. Upper limb motor and sensory impairments in children with hemiplegic cerebral palsy. Can they be measured reliably? Disabil Rehabil. 2010 32(5):409-16. doi:10.3109/09638280903171469.

50. Wilton JC. Hand splinting: Principles of design and fabrication. Perth: Success Print; 2004.

51. House JH, Gwathmey FW, Fidler MO. A dynamic approach to the thumb-inpalm deformity in cerebral palsy. Evaluation and results in fifty-six patients. J Bone Joint Surg. 1981;63(2):216-25.

52. McConnell K, Johnston L, Kerr C. Upper limb function and deformity in cerebral palsy: A review of classification systems. Dev Med Child Neurol. 2011;53(9):799-805. doi:10.1111/j.1469-8749.2011.03953.x.

53. Haley SM, Coster WJ, Dumas HM, Fragala-Pinkham MA, Kramer J, Ni P, et al. Accuracy and precision of the Pediatric Evaluation of Disability Inventory computer-adaptive tests (PEDI-CAT). Dev Med Child Neurol. 2011;53(12): 1100-6. doi:10.1111/j.1469-8749.2011.04107.x.

54. Arnould C, Penta M, Renders A, Thonnard JL. ABILHAND-Kids: A measure of manual ability in children with cerebral palsy. Neurol. 2004;63(6):1045-52. doi:10.1212/01.WNL.0000138423.77640.37.

55. Mathiowetz V, Federman S, Wiemer D. Box and Blocks Test of Manual Dexterity: Norms for 6-19 year olds. Can J Occup Therap. 1985;52(5):241-5. doi:10.1177/000841748505200505.

56. Jongbloed-Pereboom M, Nijhuis-Van Der Sanden MWG, Steenbergen B. Norm scores of the box and block test for children ages 3-10 years. Am J Occup Therap. 2013;67(3):312-8. doi:10.5014/ajot.2013.006643.

57. Koman LA, Williams RMM, Evans PJ, Richardson R, Naughton MJ, Passmore $L$, et al. Quantification of upper extremity function and range of motion in children with cerebral palsy. Dev Med Child Neurol. 2008;50(12):910-7. doi: 10.1111/j.1469-8749.2008.03098.X

58. Geerdink Y, Lindeboom R, de Wolf S, Steenbergen B, Geurts ACH, Aarts PB. Assessment of upper limb capacity in children with unilateral cerebral palsy: construct validity of a Rasch-reduced Modified House Classification. Dev Med Child Neurol. 2014:6:580-6. doi:10.1111/dmcn.12395.

59. Coster W, Bedell G, Law M, Khetani MA, Teplicky R, Liljenquist K, et al. Psychometric evaluation of the Participation and Environment Measure for Children and Youth. Dev Med Child Neurol. 2011;53(11):1030-7. doi:10.1111/j.1469-8749.2011.04094.x.

60. Davis E, Mackinnon A, Davern M, Boyd R, Bohanna I, Waters E, et al. Description and psychometric properties of the CP QOL-Teen: A quality of life questionnaire for adolescents with cerebral palsy. Res Dev Disabil. 2013; 34(1):344-52. doi:10.1016/j.ridd.2012.08.018.

61. Waters E, Davis E, Mackinnon A, Boyd R, Graham HK, Kai Lo S, et al. Psychometric properties of the quality of life questionnaire for children with CP. Dev Med Child Neurol. 2007:49(1):49-55. doi:10.1017/S0012162207000126.x

62. Ratcliffe J, Stevens K, Flynn T, Brazier J, Sawyer M. An assessment of the construct validity of the CHU9D in the Australian adolescent general population. Qual Life Res. 2012;21(4):717-25. doi:10.1007/s11136-011-9971-y. 